\title{
XXX. Ueber den grünen Apatit von Malmberget in Schweden.
}

\author{
Von \\ K. Zimányi in Budapest 1).
}

(Hierzu Tafel XIV.)

Auf meiner Studienreise im Sommer des Jahres 1902 besichtigte ich auch die mineralogische Sammlung des >Naturhistor. Riksmuseum \& zu Stockholm, wo mich Herr Dr. Gustav Lindstrüm auf die schönen schwedischen Vorkommen und Unica aufmerksam machte. Den im Folgenden zu beschreibenden grünen Apatit sah ich auch zuerst in der Stockholmer Sammlung als lose Krystalle. Die prächtige Farbe, die vollkommene Durchsichtigkeit und der Glanz der Krystalle mit ihrer schönen Ausbildung erregte mein besonderes Interesse. Der Fundort ist Gellivara in Norbotten, ohne nähere Bezeichnung. Der grüsste Krystall war etwa $4 \mathrm{~cm}$ hoch und $3 \mathrm{~cm}$ breit, die Hauptformen das Prisma mit der zugehürigen.Pyramide und die Basis. Die Krystalle waren kurz- oder langprismatisch, meistens nur an einem Ende ausgebildet.

Die berühmten Eisensteingruben sind etwa $8-10 \mathrm{~km}$ nordüstlich von Gellivara in den Bergcolonien Malmberget und Koskullskulle. An beiden Orten besuchte ich einige der Gruben und sammelte Mineralien für das ungarische Nationalmuseum. Ich betrachte es für meine angenehme Pflicht, auch an dieser Stelle den Herren Disponenten Nils. W. Bosaeus und Ottokar Koniakowski zu danken, dass sie mir gestatteten die Gruben zu besichtigen und daselbst zu sammeln, sowie dem Ilerrn Bergingenieur Axel Dellwik und Herrn C. J. P. Bergström für ihre freundliche Aufklärungen und Irührung.

1) Aus dem ungarischen Original (Annales Historico - Naturales Musei Nationalis Hungarici 1904, 2, 272) vom Verfasser mitgetheilt. 
In den Gebirgen von Gellivara ist der Apatit sehr verbreitet ${ }^{1}$ ), er findet sich nach $\mathrm{Hj}$. Lundbohm zusammen mit dem Eisenerz, als Gemengtheil des »Skarns « und Gneisses, endlich auch in Pegmatilgängen. Im Eisenerz, welches hauptsächlich Magneteisenstein ist, kommt der Apatit kürnig vor, zuweilen wechseln Streifen des Magnetits mit solchen des Apatits ab. Gewöhnlich enthält das Eisenerz 0,06\% Apatit, welcher jedoch an manchen Stellen auch auf $18 \%$ steigt.

Den grüssten Theil des untersuchten Materials verdanke ich der Güte des Herrn Bergingenieur A. Dellwik, der mehrere lose Krystalle und im Laufe des verflossenen Winters noch eine kleine, sehr lehrreiche Stufe dem ungarischen Nationalmuseum schenkte; auf mein Ersuchen sandte mir ${ }^{\circ}$ Herr Prof. Hj. Sjögren, Intendant des Stockholmer Riksmuseums, aus der mineralogischen Sammlung dieses Instituts vier Krystalle zur Untersuchung, sowie Herr Disponent H. V. Tiberg in Långbanshyttan aus seiner schünen Privatsammlung einen Krystall ebenfalls zur Untersuchung. Allen diesen Herren bin ich für ihre Güte aufrichtigen Dank schuldig. Drei von den Krystallen aus der Sammlung des Rikmuseums habe ich gemessen und an zweien auch die Brechungsexponenlen bestimmt; den Krystall des Herrn Tiberg hatte ich ebenfalls geometrisch und optisch untersucht.

Wie ich aus mündlicher Mittheilung des Herrn Bergstrüm und einem Schreiben des Herrn Dellwik erfuhr, kamen die Apatitkrystalle in der "Kung Oskar grufva* vor; ich habe in dieser Grube nur lichtgrünen und weissen kürnigen Apatit gesammelt, derselbe findet sich im Magnetit.

Nach der brieflichen Mittheilung des Hern Dellwik kommen die Apatitkrystalle in Drusenräumen gewöhnlich nahe der Oberfläche vor. An der eben erwähnten, kleinen Stufe sind die Enden der Krystalle beinahe

1) Die wichtigste Literatur über das Vorkommen les Apatits von Gellivạa: Th. Nordiström, A. Sjögren och Hj. Lundbohm, Betảnkanden afgina af den för undersökning af Apatittilgångar i Norbotten tilsatta komission. Slockholm 1890. Ref. N. Jahrb. f. Min. u.s. w. 1893, 2, 63.

Hj. Lundbohin, Apatitförekomster i Gellivara malmberg och kringlinggande trakt. Sveriges geol. Undersök. 1890, Serie C, Nr. 111.

0. Torrel, A patitförekomsterna i Norrbottenslïn. Geol. Füren. Fürh. 1890, 12, 49. Ref. N. Jahrb. f. Miner. u. s. w. 1893, 2, 63.

Hj. Lundbohm, Om Gellivara malmberg och apatilundersökingarna derstides. Geol. Fören. För. 1891, 13, 10. Ref. N. Jahrb. f. Min. U. S. w. 1893, 2, 64 und 269.

Hj. Lu ndboh th, Apatitförkomster i Norrbottens malmberg. Sveriges geol. Undersök. 1892, Ser. C, Nr. 127. Ref. N. Jahrb. f. Min. u. s. w. 1897, 9, 85.

F. Svenonius, Om berggrunden i Norrbottenslän och utsigterna till brytvärda apatitförekomster derstădes. Sveriges geol. Undersök. 1892, Ser. C, Nr. 126. Ref. N. Jahrb. f. Miner. u. s. w. 1897, 2, 8.i.

F. Svenonius, Torskingsresor i Kvikkjokksfälltrakter åren $1 \$ 92$ och 1893 med särskild hänsyn till apatitförekomster. Sveriges geol. Undersök. 1895, Ser. C, Nr. 146. 
alle abgebrochen; entweder ist das freie Ende ausgebildet, oder sind sie in ihrer ganzen Länge mit den Prismenflichen angewachsen und die terminalen Flächen kommen nicht zur Ausbildung. Die Farbe des $\Lambda$ patits ist brüunlich oder grünlichgelb, seine Begleitmineralien sind: Quarz, Albit, Epidot, Amphibol, Flusspath, Magnetit, Hämatit, Chalkopyrit, und an der unteren Seite der Stufe ein rüthlicher, schon etwas verwitterter Feldspath. Der Quarz von schmutzig weisser oder grauer Farbe zeigt die gewühnlichsten Formen $(m, r, z)$. Die kleinen $(0,5-1 \mathrm{~mm})$ dicktafeligen Krystalle des Albits sind Zwillinge nach $b(010)$, er ist farblos, weiss oder gelblichroth, nach den annähernden Messungen sind die Formen $b\{010\}$, $e\{001\}, M\{1 \overline{1} 0\}$ und $\approx\{1 \overline{3} 0\}$. Der Amphibol ist schwarzgrün, nur in dünnen Spaltungsblättchen durchsichtig, an einem solchen wurde der Prismenwinkel zu 55025'-55045' gemessen; auf (110) ist der Auslüschungswinkel 14:1.0, der Pleochroïsmus war nicht stark. Um den Amphibol, mit demselben theilweise verwachsen ist ein grünlichgraues, dünnfaseriges, asbestähnliches Mineral. Der Epidot bildet ein dünnstengeliges, pistaziengrünes Aggregat, Krystallfächen sind nicht unterscheidbar. Der Flussspath hat graulichviolette Farbe und ist als spätere Bildung auf dem $\Lambda$ patit aufgewachsen. Die gerundeten Körner und Krystalle des Magnetits sind zuweilen bläulich oder bräunlich angelaufen. Der Hämatit ist entweder auf die Quarzkrystalle gewachsen in Form von radialfürmigen Kügelchen, oder er sitzt als Eisenglimmer auf Quarz, Epidot, Flusspath und Apatit, die Schüppchen sind theilweise schon sehr dünn und roth durchscheinend. Nicht selten findet man in den Apatitkrystallen Ilämatilschüppchen als Einschluss, einzelne mit regelmässig sechsseitiger Umgrenzung; im durchfallenden Lichte tritt die rothe Farbe des Il̈̈matits sehr schün von der gelblichgrünen des Apatits hervor. Die durch IIerauslüsen separirten Schüppchen zeigen in Canadabalsam eingebeltet die starke Lichtbrechung und bei oberer Beleuchtung den eigenthümlichen Glanz. Manche Hümatitschüppchen sind parallel gelagert der Prismenflächen des Apatits. Die meistens gelblichgrünen Krystalle sind von verschiedener Farbennüance, man kann einen stufenweisen Uebergang von den mehr gelben zu den saftgrünen erkennen; es kommen jedoch entschieden gelbe und sehr blassgrüne auch vor, diese letzteren erinnern an die Farbe des Aquamarins, die theilweise oder gänzlich undurchsichtigen sind schon mehr gelblichbraun. Zuweilen zeigt ein und derselbe Krystall verschiedene Färbung, wobei jedoch der Farbenunterschied nicht so auffallend ist, wie man denselben bei manchen Turmalinen beobachten kann; das eine Ende des Krystalles ist gelb oder gelblichgrün, das andere Ende blassgrün. Die beiden Farben haben keine scharfen Grenzen, sondern gehen unregelmüssig in einander übrig.

Die gemessenen Krystalle haben eine Lünge von $5-22 \mathrm{~mm}$, ihre Dicke ist 2-11 mm; cinzelne sind kurzprismatisch, kaum länger als breit (1., 4., 
5. und 8. Krystall), bei anderen übertrifft die Länge drei- bis viermal die Breite des Krystalles (9., 10. und 11. Krystall).

An den gemessenen zwölf Krystallen wurden folgende 15 Formen beobachtet; von diesen sind die mit einem ${ }^{*}$ bezeichneten für den Apatit neu:

\begin{tabular}{|c|c|}
\hline$e\{0001\}$ & $\varepsilon\{30 \overline{3} 4\}$ \\
\hline$m\{10 T 0\}$ & $*^{*} g\{70 \overline{7} 9\}$ \\
\hline$a\{11 \overline{2} 0\}$ & $*_{j}\{70 \overline{7} 8\}$ \\
\hline$o\{10 T 3\}$ & $x\{10 \pi 1\}$ \\
\hline$r\{10 T 2\}$ & $y\{20 \overline{2} 1\}$ \\
\hline${ }^{*} e\{7.0 . \overline{7} .11\}$ & $s\{1 \mid \overline{2} 1\}$ \\
\hline${ }^{*} f\{20 \overline{2} 3\}$ & $\mu\{21 \overline{3} 1\}$ \\
\hline
\end{tabular}

Die vorherrschende Form ist immer das Prisma $m$, an dem ausgebildeten einem Ende der Krystalle sind entwickelt $x$ und $c$, bald die erstere, bald die letztere Form mit grösseren Flächen. Die übrigen Formen sind so untergeordnet, dass sie den Habitus der Krystalle nicht ändern; die neuen schmalen Flächen erscheinen zuweilen auch als Begrenzung der Furchen auf $x\{10 \overline{1} 1\}$. An den grossen Krystallen sind die Flächen von $x$ zwar glänzend, aber uneben und liefern gestürte Reflexe. Einzelne Kanten von $m\{10 \bar{T} 0\}$ werden abgestumpft durch die schmalen Flächen von $a\{11 \overline{2} 0\}$, welche vertical gestreift sind; die Flächen der Zone [10T0:01T1 $=0 \pi 1]$ sind zuweilen auch fein gerieft in der Richtung der Zonenaxe, und auch schwach gekrümmt. $O b$ diese Streifungen Wachsthumserscheinungen sind oder von nachträglicher Aetzung herrühren, konnte ich nicht mit Sicherheit entscheiden. Zwischen $\mu\{2 \mid \overline{3} 1\}$ und $s\{1 \mid \overline{2} 1\}$ findet man nicht selten haarschmale, glänzende Streifen, welche schwach reflectiren und unbestāndige Neigung haben; die abgeleiteten Indices sind sehr hoch und complicirt. An den grüsseren Flächen von \{10T1\} sieht man mehrmals Vertiefungen, welche unregelmässig hegrenzt sind, deren Boden jedoch auch von $x\{10 T 1\}$ abgeschlossen ist. Nicht selten haben die Krystalle in Folge der ungleichen Grösse der $\{10 T 1\}$-Flächen monosymmetrischen Habitus (Fig. 1, 4 und 5), und die untergeordneten Formen sind mit unvollkommener Flächenzahl entwickelt.

Die Prismenflächen liegen meistens nicht exact in der Zone und ihre Neigung zu der Basis differirt gewöhnlich um einige Minuten von $90^{\circ}$ (die

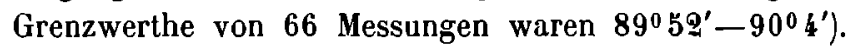

Die Flächen spiegeln gut, theilweise sogar sehr gut, dennoch differiren die analogen Winkel an den gemessenen Krystallen um $9^{\prime}-12^{\prime}$. Bekannterweise variiren zuweilen die Neigungen auch bei den sehr gut reflectirenden Flächen, selbst bei ein und demselben Krystall; die Abweichungen sind zwar gering, doch kann man sie nicht blos Beobachtungsfehlern und 
der Ungenauigkeit der Goniometer zuschreiben. Natürlicherweise darf man nicht aus diesen, einige Minuten betragenden Differenzen auf eine geringere Symmetrie oder auf die Ungültigkeit eines der Hauptgesetze der Krystallographie folgern.

Neuestens hatte Herr $\Lambda$. Miersi) an Krystallen verschiedener Salze die Veränderlichkeit der Winkel beobachtet; in einer früheren $A r b e i t^{2}$ ) stellte er genaue Messungen an ausgezeichneten Krystallen von Pyrargyrit und Proustit an, um die Neigung der Rhomboëderflächen zu ermitteln; die erhaltenen Resultate sind folgende:

$$
\begin{array}{cc}
\text { Pyrargyrit: } & \multicolumn{2}{c}{\text { Proustit: }} \\
(10 \bar{\top}):(\bar{T} 101)=71017^{\prime}-71^{0} 23^{\prime} & 72^{\prime} 8^{\prime}-72018^{\prime} \\
(01 T 2):(\bar{T} 012)=42 \quad 1-42 \quad 6 & 4243-4247
\end{array}
$$

Verschiedene Forscher erhiellen an Krystallen desselben Fundortes, obwohl ihnen gutes Material zur Verfügung stand, nicht dieselben Winkelwerthe; so wurde z. B. an dem Apatil von Jumilla gemessen:

$$
\begin{aligned}
& \text { Rose }{ }^{3 ;} \text { : } \\
& (10 \overline{1} 1):(01 T 1)=37^{\circ} 39^{\prime} 45^{\prime \prime} \\
& \text { (10T1) }:(T 011)=8028 \quad 0 \\
& \text { v. Kokscharow5: } \\
& \text { Schrau( } 4) \text { : } \\
& 37^{\circ} 37^{\prime} 10^{\prime \prime}-37^{\circ} 40^{\prime} 20^{\prime \prime} \\
& \begin{array}{llllll}
80 & 21 & 45 & -80 & 25 & 5
\end{array} \\
& \text { Baumhauer6): } \\
& (10 T 1):(01 T 1)=37040^{\prime} 30^{\prime \prime}-37^{\circ} 44^{\prime} 0^{\prime \prime} \quad 37^{0} 36^{\prime} 30^{\prime \prime}-37042^{\prime} 0^{\prime \prime} \text {. }
\end{aligned}
$$

Wie weit die analogen Winkel an ein und demselben Krystalle manchmal differiren, ersieht man aus den genauen Messungen v. Kokscharow's und Strüver's, wclche an dem Apatit von Kirjabinsk und an einem Spinelloktaëder ausgeführt wurden.

$$
\begin{aligned}
& \text { v. Kokscharowit): } \\
& s: s^{\prime}=(11 \overline{2} 1):\left(2 T^{\top} T 1\right)=48^{\circ} 4 \mathrm{I}^{\prime} 40^{\prime \prime}-48^{\circ} 47^{\prime} 30^{\prime \prime} \quad \mathrm{5}^{\prime} 50 \\
& s: x=(11 \overline{2} 1):(10 \overline{1} 1)=264 \overline{5} 50-26 \quad 52 \quad 30 \quad 440 \\
& r: e=\left(10 \bar{\top}^{2}\right):(0001)=22 \quad 47 \quad 10-225 i 40 \quad 730 \\
& \text { Strüver } 8 \text { ): } \\
& \text { (111): }(1 T 1)=70^{\circ} 29^{\prime} \quad 5^{\prime \prime}-70^{\circ} 33^{\prime} 40^{\prime \prime} \quad 4^{\prime} 35^{\prime \prime}
\end{aligned}
$$

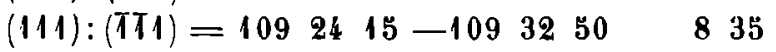

Das Mittel der gemessenen besten Winkel und der aus diesen abgeleiteten Axenlängen steht am nächsten dem der $\Lambda$ patite von Kirjabinsk und

1) Philos. Transact. of the Royal Soc. of London 1903, 202, Scr. A, 459. Diese Zeitschr. 1904, 39, 220 .

2) Diese Zeitschr. 1889, 15, 138.

3) Pogg. Ann. 1827, 9, 197.

4) Sitzungsber. d. Wien. $\Lambda$ kad. 1870,69 (11), 745.

5) Materialien z. Miner. Ilusslands 1854-57, 2, 66.

6) Diese Zeitschr. 1891, 18, 29.

7) Material. z. Miner. Russlands 1866, 5, sechster Anhang zum Apatit 90, 91 u. 98,

8) Diese Zeitschr. 1878, 2, 480. 
Jumilla 1). Die Axenlänge berechnete ich aus den Winkeln (10T1):(0001) und $(10 \bar{T} 1):(01 \bar{T} 1)$, und da dieselbe unbedeutend differirt, nahm ich aus beiden das Mittel:

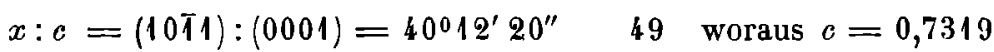

$$
\begin{aligned}
& x: x^{\prime}=(10 \bar{T} 1):(01 \overline{1} 1)=373955 \quad 29 \quad-\quad c=0,7321
\end{aligned}
$$

aus welchen das Mittel:

$$
c=0,7320 \text {. }
$$

Nach den vorläufigen Untersuchungen des IIerrn J. Loczka haben wir es mit einem Fluorapatit zu thun, welcher etwas mehr als $0,5 \% \mathrm{Cl}$ enthält.

Folgende Tabelle zeigt die an zwölf Krystallen gemessenen Normalwinkel und die aus dem Mittelwerthe der Axenlänge berechneten Winkel, Kr. bezieht sich auf die Zahl der gemessenen Krystalle, $n$ auf diejenige der

\begin{tabular}{|c|c|c|c|c|c|c|c|c|c|}
\hline$c: \sigma=(0001)$ & $:(10 \overline{1} 3)=$ & & $\begin{array}{r}\text { Gren } \\
033^{\prime}\end{array}$ & $\begin{array}{l}\text { werthe: } \\
\text { 1. }-15^{0}\end{array}$ & $58^{\prime} \mathrm{ct}$ & kr. & & $\begin{array}{l}\text { Gemessen: } \\
15^{0} 45^{\prime} \mathrm{ca}\end{array}$ & $\begin{array}{l}\text { Berechne } \\
15^{0} 44^{\prime}\end{array}$ \\
\hline$: r=$ & $:(10 \pi 2)$ & 22 & 38 & -23 & 3 & 7 & 23 & 2247 & 22343 \\
\hline$: e=$ & $:(7.0 . \overline{7} .11$ & ) 98 & 10 & -28 & 21 & 3 & 3 & 2814 & 28165 \\
\hline$: f=$ & $:(20 \overline{\mathcal{Q}} 3)$ & & & & & 1 & 1 & $29 \quad 26$ & $29 \quad 24$ \\
\hline$: \varepsilon=$ & $:(30 \overline{34})$ & & & & & 1 & 1 & 3229 & 32221 \\
\hline$: g=$ & $:(7079)$ & 33 & 11 & -33 & $35 \mathrm{c}$ & a. 2 & 2 & $33 \quad 11$ & $\begin{array}{lll}33 & 19 & 1\end{array}$ \\
\hline$: j=$ & $:(70 \overline{7} 8)$ & 36 & 34 & -36 & 53 & 3 & 4 & 3645 & $\begin{array}{lll}36 & 29 & 1\end{array}$ \\
\hline$: x=$ & $:(10 T 1)$ & 40 & 7 & -40 & 17 & 12 & 49 & $40 \quad 12$ & 40122 \\
\hline$: y=$ & $:(20 \overline{2} 1)$ & 59 & 10 & $-\quad 59$ & 39 & 11 & 38 & $59 \quad 21$ & $\begin{array}{lll}59 & 23 & 3\end{array}$ \\
\hline$: m=$ & $:(10 \overline{1} 0)$ & 89 & 53 & -90 & 4 & 12 & 66 & 8959 & 90.0 \\
\hline$: s=$ & $:(1 \mid \overline{2} 1)$ & & & & & 1 & 1 & 5ั5 37 & $\begin{array}{lll}55 & 39 & 5\end{array}$ \\
\hline$m: n=(10 T 0)$ & $:(31+1)$ & 22 & 12 & --23 & 22 & 6 & 8 & 2224 & 22434 \\
\hline$: \mu=$ & $:(21 \overline{3} 1)$ & 30 & 9 & -30 & 37 & 8 & 28 & $30 \quad 19$ & $\begin{array}{ll}30 & 23 \\
2\end{array}$ \\
\hline$: s=$ & $:\{|| \bar{Q} \mid\}$ & 44 & 7 & -44 & 32 & 10 & 53 & 4421 & 43204 \\
\hline$: x^{\prime}=$ & $:(01 \bar{T} 1)$ & 71 & 4 & -71 & 13 & 12 & 57 & 719 & $71 \quad 101$ \\
\hline$x: x^{\prime}=(10 \overline{1})$ & $:(01 T 1)$ & 37 & $3 \pi$ & -37 & 49 & 11 & 29 & $37 \quad 40$ & $\begin{array}{lll}37 & 39 & 3\end{array}$ \\
\hline$a: m=(11 \overline{2} 0)$ & $:(10 \overline{0} 0)$ & 29 & 57 & -30 & 3 & 1 & 6 & 300 & $30 \quad 0$ \\
\hline
\end{tabular}
Kanten.

Die Combinationen der gemessenen Krystalle sind:

1. Krystall: $m, c, x$ (Fig. 1).

5. - $\quad m, x, c, y$ (Fig. 2).

9. - $\quad m, x, s, y, c, \mu$ (Fig. 3).

10. - $\quad m, x, c, s, y, j$.

8. - $\quad m, x, c, s, a, r, y$ (Fig. 4).

11. - $m, x, c, s, y, r, \mu$.

1) Diese Zeitschr. 1902, 36, 447, 
3. Krystall: $m, x, c, y, s, \mu, n, r$.

4. - $m, x, c, s, y, \mu, r, n$.

6. - $\quad m, c, x, s, y, a, \mu, n$.

2. - $\quad m, x, c, y, s, a, \mu, n, r, \sigma, e$ (Fig. 5).

12. - $\quad m, x, c, y, a, s, \mu, \sigma, r, e, g, j, n$ (Fig. 6).

7. - $\quad m, x, e, y, s, \mu, n, r, e, t, \varepsilon, g, j$.

Von den zwölf gemessenen Krystallen bestimmte ich an vier auch die Brechungsindices, es waren dic lebhaft grünen Krystalle Nr. 9 (Fig. 3) und Nr. 10, ferner die grünlichgelben Nr. 1 (Fig. 1) und Nr. 8 (Fig. 4); diese vier Krystalle bezeichnete ich bei der optischen Bestimmung mit den Nummern $15,16,10$ und 13.

Schon mehrfach wurden die Brechungsindices verschiedener Apatite bestimmt, aber nur die Untersuchungen Heusser's1) und Schrauf's') erstreckten sich auf verschiedene Wellenlängen des Spectrums; neuerer Zeit hat J. E. Wolf ${ }^{3}$ ) dic Lichtbrechungsverhältnisse des schönen $\Lambda$ patits von Minot (Maine) für $N a$ - und $L i$-Licht bestimmt.

Von deın zu meiner Verfügung gestandenen Material wählte ich das entsprechendste zu den optischen Bestimmungen aus; um zu erfahren, ob mit der liärbung auch die Lichtbrechung einc andere ist, führte ich die Beobachtungen auch an Krystallen von verschiedener Farbe aus. Dass mit der Verschiedenheit und Intensitit der Farbe auch die Lichtbrechungsverhältnisse sich oft ändern, dafür können als Beispicle dienen die Turmaline ${ }^{4}$ ), Diopside ${ }^{5}$ ), Berylle ${ }^{6}$ ), Korunde ${ }^{7}$ ), Quarze ${ }^{8}$ ) und Flusspathe ${ }^{9}$. Die Frage, bis

1) Pogg. Ann. 1852, 87, 467.

2) Sitzungsber. d. Wien. $\Lambda$ kad. $1860,42,114$.

3) Diese Zeitschr. 1902, 36, 445.

4) E. A. Wülfing, Ueber einige krşstallographische Constanten des Turmalins u. s. w., Stultgart 1900. Ref. diese Zeitschr. 1902, 36, з38.

5) E. A. W ülfing, Beiträge zur kenntniss der Pyroxenfamilie in chemischer und optischer Beziehung. Habilitationsschrift, Tübingen 1891. Ref. diese Zeitschr. 1894, 23, 297. - Al. Schmidl, Daten zur genauen Kenntniss einiger Mineralien der Pyroxengruppe. Diese Zeitschr. 1893, 21, 1.

6) C. Hintze, Handbuch der Mineralogie 1900, 2, $1 \geq 73$ und diese Zeilschr. 1902, 36, $98-99$.

7) G. Melczer, lieber einige krystallographische Constanten des Korund. Diese Zcitschr. 1902, 35, 561 .

8) H. Dufet, Mesures comparatives de l'indice de differents quartz. Bull. d. l. soc. fr. d. Min. 1890, 13, 271. Ref. diese Zeitschr. 1893, 21, 281. - C. Hlawatsch, Ueber den Brechungsexponenten einiger pigmentirter Mineralien. Diese Zeitschr. 1897, 27, 606 .

9) F. Kohluausch, Die Ermitlelung von Lichtbrechungsverhältnissen fesler Körper durch Totalrellexion. Diese Zeitschr. 1878, 2, 101. - C. Hlawatsch, a. a. O. II. Dudenhausen, Optische Cntersuchungen an Flussspath und Steinsalz. N, Jahrb, f. Miner, U. s. w. 1904, 1, 8. 
zu welchem Grade die dilute Fărbung die Aenderung der Brechungsexponenten beeinflusst, oder ob dieselbe nur Folge der veränderten chemischen Zusammensetzung ist, scheint noch nicht endgültig gelöst zu sein; jedenfalls sind noch ausführlichere Untersuchungen an genügendem und tadellosem Minerale erwünscht, bei welchen auch die $\Lambda$ bsorptionserscheinungen ${ }^{1}$ ) erfołgreich angewendet werden künnten. Dudenhausen hat an verschieden gefirbten Fluoriten und an dem Steinsalz Bestimmungen der Brechungscxponenten ausgeführt; da er verhältnissmässig geringe Unterschiede beobachtete, kommt er zu dem Schlusse ${ }^{2}$ ): »dass die Unterschiede in den Brechungsexponenten hauptsächlich nur auf Beobachtungsfehlern beruhen, die Färbung dagegen nur einen ganz unwesentlichen Einfluss dabei ausübt.

Die Erfahrungen Wolff's ${ }^{3}$ ) an dem Apatit von Minot scheinen auch zu zeigen, dass die dilute lärbung kaum einen Einfluss auf die Licht- und Doppelbrechung der Mineralien hat, da der ursprünglich violette $\Lambda$ patitkrystall nach der durch Erhilzen erfolgten Entfärbung dieselbe Lichtbrechung hatte; es wäre wünschenswerth, bei dilut gefärbten Mineralien die Lichtbrechung nicht blos an verschieden gefürbten Krystallen zu bestimmen, sondern auch die Intensität der Farbe zu berücksichtigen und die Beobachtungen müglicher Weise an mehreren Krystallen auszuführen, wie dies Melczer an dem Korund unlernahm.

Ich benulzte zu meinen Messungen das Fuess'sche Goniometer Modell Nr. II; die Beobachtungen führte ich meistens mit dem stark vergrössernden Fernrohr $c$ aus, dessen Ramsden'sches Ocular eine etwa sechsfache Vergrösserung hat. Bei $L i$ - und $T l$-Licht benutzte ich das schwächere $b$-Fernrohr ${ }^{4}$ ) bei den Krystallen Nr. 1, 9, 3, 10, 11, 12 und 14, bei den Krystallen Nr. 15 und 16 das Fernrohr mit dem Ramsdenschen Oculare. Nls Signal gebrauchte ich den Websky'schen Spalt.

In zehn Krystallen bestimmte ich die Brechungsindices durch Messung des Winkels der Minimalabweichung ( $\delta$ ); bei sechs Krystallen ( $\mathrm{Nr} .1,9,3,10$, $11,12)$ beziehen sich die Bestimmungen auf fünf verschiedene Wellenlïngen, und zwar auf $\mathrm{Li}$-, $\mathrm{Na}$-, Tl-Licht und auf die beiden Linien des Wasserstoffspectrums $H_{\alpha}$ und $H_{\beta}$; bei drei Krystallen (Nr. 14, 15 und 16) auf $L i$, $\mathrm{Na}$ - und $\mathrm{Tl}$-Licht; endlich bei dem Krystalle $\mathrm{Nr} .13$ nur auf $\mathrm{Na}$-Licht.

Zur Messung dienten mir nur die natürlichen Flächen des Prismas $m\{10 T 0\}$; ausserdem bildete an zwei Krystallen (Nr. 1, 13) das brechende Prisma noch eine Prismenfläche und eine hintere Pyramidenfläche $\alpha=$ $(10 \overline{1} 0):(\overline{1} 011)$. Nur die glattesten Partien der gut reflectirenden Flächen

1) $\Lambda . \mathrm{Nabl}$, Leber färbende Bestandtheile des $\Lambda$ methysten, Citrins und gebrannten Amethysten. Sitzungsber. đ. Akad. Wien 1899,108 , Ablh. $11 \mathrm{~b}, 48$. Ref. diese Zeitschrift $1900,33,632$.

2! a. a. 0. S. 29.

3) Diese Zeitschr. 1902, 36, $44 \mathrm{j}$.

4) C. Leiss, Die optischen Instrumente der Firma R, Fuess. Leipzig $1899,124$. 
wurden benutzt, die übrigen Theile mit schwarzer Farbe verdeckt; die freigelassene Partie der abgeblendeten Prismenflāchen hatte eine Breite von $1 \frac{1}{2}-2 \frac{1}{2} \mathrm{~mm}$.

Jede Beobachtung wiederholte ich mehrmals, der Lichtstrahl fiel bald auf die eine, bald auf die andere Prismenfläche, abgelesen wurde immer auf anderen Theilen des Limbus.

Die mitgetheilten Mittelwerthe der minimalen Deviation sind auf 5-5 Secunden abgerundet. War das abgelenkte Bild des Websky'schen Spaltes intensiv und scharf, so war die Abweichung der einzelnen Ablesungen $0^{\prime} 15^{\prime \prime}-1^{\prime} 15^{\prime \prime}$; bei den meisten meiner Bestimmungen hatte die Genauigkeit diesen Grad; bei einem kleineren Theile waren diese Differenzen 1'25"-2' 25 ", was die Brechungsexponenten mit $\pm 0,0001-0,0002$ beeinflusst; nur bei drei Krystallen in fünf Füllen differirten die einzelnen $\Lambda$ blesungen bei grünem und blauem Lichte von 2' 25"-3' 0", in Folge dessen die Genauigkeit der Brechungsexponenten noch geringer war als $\pm 0,0002$.

Wie man aus der weiter unten mitgetheilten tabellarischen Zusammenstellung sieht, ist die Brechung einerseits für $o_{L i}$ und $e_{N a}$, andererseits für $o_{N a}$ und $e_{T l}$ nahe dieselbe, in Folge dessen diese abgelenkte Strahlen sich theilweise decken. Bei der grüssten Sorgfalt war es mir nicht immer müglich, dass $N a$-Dämpfe das grüne oder rothe Licht nicht stören, in solchen Fällen wir auch schwach die $\mathrm{Na}$-Linie sichtbar, und ich benutzte zur Auslöschung derselben das Nicol'sche Prisma.

Die Intensität der zwei abgelenkten Strahlen war verschieden, der extraordinäre war entsprechend der stärkeren Absorption immer schwächer. Dies kann man gut beobachten, wenn man die Stärke der Lichtquelle bis zu einem gewissen Grade vermindert, da sieht man nur noch den ordinären Strahl. Die Absorption ist um so stärker, je intensiver die Farbe des Krystalles ist, so ist z. B. dieselbe bei dem grünlichgelben Krystalle Nr. 13 (Fig. 4) sehr auffallend, hingegen bei den blassgrünen (Nr. 14) sehr schwach; die vom Lichte durchlaufene Schicht beträgt im Mittel bei dem ersteren Prisma 5-6 $\mathrm{mm}$, bei dem letzteren 1-2 $\mathrm{mm}$. Bei den zwei Krystallen, wo ich die Brechungsexponenten durch ein Prisma, gebildet von (10T1) und (T010), bestimmte, wo also der ausserordentliche Strahl zu dem ordentlichen näher lag, war die Lichtintensität der zwei abgelenkten Bilder kaum bemerkbar.

Die Farbe der Krystalle war gelb, lebhaft grün, grünlichgelb und blassgrün. Die Farbe des gelben (Nr. 2) Krystalles erinnert an jene von Jumilla, nur ist der von Malmberget dunkler; die lebhaft grün gefärbten (Nr. 9, $15,16)$ haben eine Nüance in das Gelbliche, die zwei letzteren sind dunkler als der erstere. Die grünlichgelben Krystalle (Nr. 3, 10, 11, 12, 13) haben gleiche Farbe, nur ist einer lichter, der andere dunkler. Blassgrün ist der Krystall Nr. 14. 
Die Resultate meiner Messungen stelle ich in der folgenden Tabelle zúsammen, auf die gleich oder ähnlich gefärbten Krystalle bezügliche Werthe folgen unmittelbar auf einander. Bei Krystall Nr. 1 und Nr. 13, bei welchen das brechende Prisma auch eine Pyramiden- und eine entgegenliegende Prismenfläche bildeten, benutzte ich zur Berechnung von $\varepsilon$ die bekannte Formel ${ }^{1}$ ):

und:

$$
\begin{gathered}
A_{0} \mathrm{e}^{4}+2 A_{1} \mathrm{e}^{2}+A_{2}=0, \text { worin: } \\
A_{0}=\sin ^{2} \tau \\
2 A_{1}=\left(\mathfrak{o}^{2}+\sin ^{2} \varrho\right. \\
\left.C^{2}+\frac{\cos ^{2} \varrho}{S^{2}}\right) \cos ^{2} \tau-\frac{1}{C^{2}}-\frac{1}{S^{2}} \\
A_{2}=-\mathfrak{v}^{2}\left(\frac{\sin ^{2} \varrho}{C^{2}}+\frac{\cos ^{2} \varrho}{S^{2}}\right) \cos ^{2} \tau+\frac{1}{C^{2} S^{2}}
\end{gathered}
$$

$$
{ }_{C}=\frac{\cos \frac{A}{2}}{\cos \frac{A+\delta_{e}}{2}}, \quad \frac{1}{S}=\frac{\sin \frac{A}{2}}{\sin \frac{A+\delta_{\rho}}{2}} .
$$

\begin{tabular}{|c|c|c|c|c|c|c|c|c|}
\hline 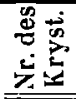 & $a$ & Lichtart & $\delta_{e}$ & $\varepsilon$ & & $\delta_{o}$ & $\boldsymbol{\omega}$ & $\omega-\varepsilon$ \\
\hline 1. & $\begin{array}{c}6009^{\prime} 30^{\prime \prime} \\
a= \\
(10 \overline{1}):(\overline{1010}) \\
49048^{\prime} 0^{\prime \prime}\end{array}$ & $\begin{array}{l}L i \\
H_{\alpha} \\
N a \\
T l \\
H_{\mu} \\
L i \\
N a \\
T l\end{array}$ & $\begin{array}{rrrr}490 & 30^{\prime} & 25^{\prime} \\
49 & 33 & 25 \\
49 & 49 & 55 \\
50 & 10 & 20 \\
50 & 32 & 40 \\
36 & 55 & 30 \\
37 & 8 & 35 \\
37 & 21 & 30\end{array}$ & $\begin{array}{l}1,6310 \\
1,6315 \\
1,6343 \\
1,6377 \\
1,6414 \\
1,6301 \\
1,6337 \\
1,6364\end{array}$ & $\begin{array}{l}490 \\
49 \\
50 \\
50 \\
50 \\
37 \\
37 \\
37\end{array}$ & $\begin{array}{rr}53^{\prime} & 25^{\prime \prime} \\
56 & 0 \\
13 & 0 \\
33 & 10 \\
56 & 35 \\
8 & 30 \\
21 & 15 \\
34 & 40\end{array}$ & $\begin{array}{l}1,6349 \\
1,6353 \\
1,6381 \\
1,6415 \\
1,6453 \\
1,6340 \\
1,6372 \\
1,6406\end{array}$ & $\begin{array}{l}0,0039 \\
0,0038 \\
0,0038 \\
0,0038 \\
0,0039 \\
0,0039 \\
0,0037 \\
0,0048\end{array}$ \\
\hline 9. & 5958 & $\begin{array}{l}L i \\
H_{\alpha} \\
N a \\
T l \\
H_{\beta}\end{array}$ & $\begin{array}{rrr}49 & 7 & 30 \\
49 & 12 & 25 \\
49 & 29 & 10 \\
49 & 47 & 45 \\
50 & 10 & 40\end{array}$ & $\begin{array}{l}1,6300 \\
1,6308 \\
1,6336 \\
1,6367 \\
1,6406\end{array}$ & $\begin{array}{l}49 \\
49 \\
49 \\
50 \\
50\end{array}$ & $\begin{array}{rr}29 & 30 \\
34 & 35 \\
52 & 5 \\
10 & 50 \\
34 & 35\end{array}$ & & $\begin{array}{l}0,0037 \\
0,0037 \\
0,0039 \\
0,0039 \\
0,0039\end{array}$ \\
\hline 15. & 130 & $\begin{array}{l}\mathrm{Li} \\
\mathrm{Na} \\
\mathrm{Tl}\end{array}$ & $\begin{array}{rrr}49 & 15 & 5 \\
49 & 35 & 10 \\
49 & 54 & 10\end{array}$ & $\begin{array}{l}1,6304 \\
1,6338 \\
1,6370\end{array}$ & $\begin{array}{l}49 \\
49 \\
50\end{array}$ & $\begin{array}{ll}7 & 40 \\
58 & 20 \\
17 & 15\end{array}$ & & $\begin{array}{l}0,0038 \\
0,0038 \\
0,0037\end{array}$ \\
\hline 16. & 60 & $\begin{array}{l}\mathrm{Li} \\
\mathrm{Na} \\
\mathrm{Tl}\end{array}$ & $\begin{array}{rrr}49 & 6 & 55 \\
49 & 27 & 15 \\
49 & 46 & 5\end{array}$ & $\begin{array}{l}1,6294 \\
1,6328 \\
1,6360\end{array}$ & $\begin{array}{l}49 \\
49 \\
50\end{array}$ & $\begin{array}{rl}27 & 10 \\
47 & 55 \\
7 & 15\end{array}$ & & \\
\hline 3. & 60 & $\begin{array}{l}L i \\
H_{a} \\
N a \\
T l \\
H_{\beta}\end{array}$ & $\begin{array}{lll}49 & 12 & 10 \\
49 & 13 & 20 \\
49 & 30 & 55 \\
49 & 50 & 20 \\
50 & 11 & 20\end{array}$ & $\begin{array}{l}1,6303 \\
1,6305 \\
1,6334 \\
1,6367 \\
1,6402\end{array}$ & $\begin{array}{l}49 \\
49 \\
49 \\
50 \\
50\end{array}$ & $\begin{array}{rr}35 & 10 \\
36 & 0 \\
54 & 10 \\
14 & 25 \\
35 & 45\end{array}$ & $\begin{array}{l}1,6341 \\
1,6343 \\
1,6373 \\
1,6406 \\
1,6442\end{array}$ & $\begin{array}{l}0,0038 \\
0,0038 \\
0,0039 \\
0,0039 \\
0,0040\end{array}$ \\
\hline
\end{tabular}

Da in unserem Falle die optische $A x e$ in einer Fläche (T010) des brechenden Prismas liegt, und zwar vertical zur brechenden Kante, so ist:

$$
\varrho=\frac{A}{2} \quad \text { und } \quad \tau=0 \text {. }
$$

1) Th. Liebisch, Physikalische Krystallographie, Leipzig 1891, 389. 


\begin{tabular}{|c|c|c|c|c|c|c|c|}
\hline 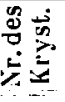 & r & Lichtart & $\delta_{e}$ & $\varepsilon$ & $\delta_{0}$ & $\omega$ & $\omega-\varepsilon$ \\
\hline 10. & $60^{0} 0^{\prime} 10^{\prime \prime}$ & $\begin{array}{l}L i \\
H_{c \iota} \\
N a \\
T l \\
H_{q}\end{array}$ & $\begin{array}{rrr}49012^{\prime} & 40^{\prime \prime} \\
49 & 15 & 5 \\
49 & 32 & 15 \\
49 & 51 & 5 \\
50 & 14 & 0\end{array}$ & $\begin{array}{l}1,6303 \\
1,6307 \\
1,6336 \\
1,6368 \\
1,6406\end{array}$ & $\begin{array}{llll}49033^{\prime} & 45^{\prime \prime} \\
49 & 36 & 53 \\
49 & 59 & 35 \\
50 & 14 & 10 \\
50 & 37 & 15\end{array}$ & $\begin{array}{l}1,6339 \\
1,6344 \\
1,6373 \\
1,6406 \\
1,6444\end{array}$ & $\begin{array}{l}0,0036 \\
0,0037 \\
0,0037 \\
0,0038 \\
0,0038\end{array}$ \\
\hline 11. & $6028 \quad 15$ & $\begin{array}{l}L i \\
H_{a} \\
N a \\
T l \\
H_{i}\end{array}$ & $\begin{array}{lll}49 & 54 & 40 \\
49 & 57 & 10 \\
50 & 14 & 20 \\
50 & 33 & 50 \\
50 & 56 & 25\end{array}$ & $\begin{array}{l}1,6305 \\
1,6309 \\
1,6338 \\
1,6370 \\
1,6406\end{array}$ & $\begin{array}{rrr}50 & 18 & 10 \\
50 & 21 & 5 \\
50 & 39 & 0 \\
50 & 58 & 35 \\
51 & 22 & 5\end{array}$ & $\begin{array}{l}1,6344 \\
1,6349 \\
1,6378 \\
1,6410 \\
1,6448\end{array}$ & $\begin{array}{l}0,0039 \\
0,0040 \\
0,0040 \\
0,0040 \\
0,0042\end{array}$ \\
\hline 12. & $\begin{array}{lll}59 & 39 & 30\end{array}$ & $\begin{array}{l}I_{i} \\
H_{a} \\
N a \\
T l \\
H_{\xi}\end{array}$ & $\begin{array}{lll}49 & 11 & 15 \\
49 & 14 & 15 \\
49 & 30 & 30 \\
49 & 50 & 15 \\
50 & 12 & 20\end{array}$ & $\begin{array}{l}1,6302 \\
1,6307 \\
1,6335 \\
1,6368 \\
1,6405\end{array}$ & $\begin{array}{lll}49 & 33 & 35 \\
49 & 36 & 30 \\
49 & 53 & 45 \\
50 & 14 & 10 \\
50 & 36 & 35\end{array}$ & $\begin{array}{l}1,6340 \\
1,6345 \\
1,6374 \\
1,6408 \\
1,6445\end{array}$ & $\begin{array}{l}0,0038 \\
0,0038 \\
0,0039 \\
0,0040 \\
0,0040\end{array}$ \\
\hline 13. & $\begin{aligned} & 60 \quad 1 \quad 20 \\
= & \left(10 T_{1}\right):\left(T_{010} 0\right) \\
= & 49045^{\prime} 40^{\prime \prime}\end{aligned}$ & $\begin{array}{l}N a \\
N a\end{array}$ & $\begin{array}{lll}49 & 27 & 25 \\
37 & 2 & 30\end{array}$ & $\begin{array}{l}1,6325 \\
1,6325\end{array}$ & $\begin{array}{lll}49 & 49 & 3 \\
37 & 14 & 55\end{array}$ & $\begin{array}{l}1,6361 \\
1,6363\end{array}$ & $\begin{array}{l}0,0036 \\
0,0098\end{array}$ \\
\hline 14. & 645 & $\begin{array}{l}I i \\
N a \\
T l\end{array}$ & $\begin{array}{lll}49 & 19 & 15 \\
49 & 39 & 20 \\
49 & 59 & 40\end{array}$ & $\begin{array}{l}1,6298 \\
1,6332 \\
1,6366\end{array}$ & $\begin{array}{rrr}49 & 41 & 30 \\
50 & 2 & 40 \\
50 & 20 & 30\end{array}$ & $\begin{array}{l}1,6336 \\
1,6371 \\
1,6404\end{array}$ & $\begin{array}{l}0,0038 \\
0,0039 \\
0,0038\end{array}$ \\
\hline
\end{tabular}

Zum Vergleiche gebe ich hier die Brechungsexponenten des spargelgrünen Apatits von Jumilla ${ }^{1)}$ und Sondalo ${ }^{2}$ ).

$$
\begin{aligned}
\text { Jumilla: } & \text { Sondalo: } \\
\omega_{D}=1,63891-1,63906 & \omega_{N a}=1,6379 \\
\varepsilon_{D}=1,63437-1,63461 & \varepsilon_{N a}=1,6349 \\
\omega_{D}-\varepsilon_{D}=0,00454-0,00445 & \omega_{N a}-\varepsilon_{N a}=0,0030
\end{aligned}
$$

Wie man aus der Tabelle sieht, ist ein kleiner Unterschied in der Lichtbrechung der einzelnen Krystalle; die schwächste Lichtbrechung besitzt der grünlichgelbe Krystall Nr. 13, die stärkste der erste Krystall.

$$
\begin{array}{ccc}
\text { hr. } 13 & \text { Kr. } 1 & \text { Unterschied: } \\
\omega_{\lambda_{a}}=1,6362 & \omega_{v_{\alpha}}=1,6381 & 0,0019 \\
\varepsilon_{V_{a}}=1,6325 & \varepsilon_{\lambda a}=1,6313 & 0,0018
\end{array}
$$

Der Unterschied ist also bei beiden Strahlen beinahe 0,002. Solche und noch grössere Unterschiede sind bei den farbigen Mineralien nicht ungewöhnlich, wie dies auch Wülfing ${ }^{3}$ ) und Melczer ${ }^{4}$ ) an den Turmalinen und Rubinen beobachteten, wobei zu bemerken ist, dass jene bei den 'Turmalinen entschieden von der chemischen Zusammensetzung abhängig ist.

1) Sitzungsber. d. Wien. Akad. $1860,42,114$.

2) Diese Zeitschr. 1902, 36, 100.

3; a. a. $0 . \quad$ i) a. a. 0 . 
In der Farbe der lebhaft grünen Krystalle (Nr. 9, 15, 16) ist kaum ein bemerkbarer Unterschied, die Lichtbrechung des Krystalles Nr. 9 und 15 ist beinahe übereinstimmend (Diff. $0,0002-0,0005$ ), hingegen ist in dieser Hinsicht bei dem Krystalle Nr. 15 und 16 der Unterschied schon grösser, nämlich $0,0010-0,0014$. Noch grösser ist die Uebereinstimmung bei den grünlichgelben Krystallen Nr. 3, 10 und 12 (Diff. 0,0001-0,0002). Die geringste Lichtbrechung besitzt der grünlichgelbe Krystall $\mathrm{Nr} .13$, der blassgrüne Nr. 14 steht beiläufig in der Mitte.

Bei dem grossen Krystalle Nr. 13 an zwei Stellen bestimmte Brechungsexponenten differiren sehr gering, die Unterschiede liegen innerhalb der Beobachtungsfehler. Bei dem viel kleineren $(3-4 \mathrm{~mm})$ gelben Krystalle Nr. 1 sind die durch die zwei Prismenflächen bestimmten Brechungsexponenten im Mittel um 0,0009 grüsser, als wenn das brechende Prisma eine Pyramiden- und eine Prismenfläche bildeten. Weder bei dem einen, noch bei dem anderen Krystalle war ein bemerkbarer Farbenunterschied an den zur Bestimmung der Brechungsexponenten benutzten Partien. Dass zuweilen noch grössere Differenzen der Brechungsexponenten an den verschiedenen Partien ein und desselben Krystalles vorkommen, wurde durch die Beobachtungen Wülfing's ${ }^{1}$ ) an den Turmalinen von Barrado Perahy (Brasilien), Paris (Maine) und Alabaschka bestätigt. Viola ${ }^{2}$ ) schnitt aus einem zimmetfarbigen Turmalinkrystalle von Elba drei Prismen, von welchen das Prisma Nr. V und Nr. VI aus knapp an einander gelegenen Stellen waren, in diesen differirten die Brechungsexponenten rund um fünf Einheiten in der dritten Decimale ( $\left.\omega_{N a}=1,61497-1,65056\right)$; diese Differenzen führt Autor nicht auf Beobachtungsfehler zurück, sondern sucht ihre Ursachen im Turmalin.

Die Stärke der Doppelbrechung ist kaum verschieden, bei verschiedenem Lichte variirt $\omega-\varepsilon=0,0034-0,0042$. Heusser ${ }^{3}$ ) zeigte an dem wasserklaren Apatit vom Zillerthal und Schrauf ${ }^{4}$ ) an dem gelben von Jumilla, dass die Doppelbrechung für rothes Licht geringer ist als für blaues; bei meinen Beobachtungen ist dies nicht so auffallend, da die Genauigkeit der Brechungsexponenten nur 上 $0,0001-0,0003$ ist, jedoch bei den Krystallen Nr. 3, 10, 11 und 12 ist es bemerkbar. Wie aus der Tabelle S. 517 oben sichtbar ist, besteht kein grosser Unterschied bezüglich der Dispersion, für beide Strahlen ist diese beinahe gleich stark.

Kraatz-Koschlau, Wöhler') und Wolff6) haben gezeigt, dass manche gefärbte Apatite durch Erhitzen farblos werden. Die Brechungs-

1) a. a. 0. S. 61, 62, Tab. XIV; S. 64, 66, Tab. XV; S. 66, 68, Tab. XVII.

2) Diese Zeitschr. 1903, 37, 135-137.

3) a. a. 0.4 4) a. a. 0.

5) Tschermak's Miner. Mitth. 1899, 18, 319. Ref. diese Zeitschr. 1900, 33, 613.

6) Diese Zeitschr. 1902, 36, 445. 


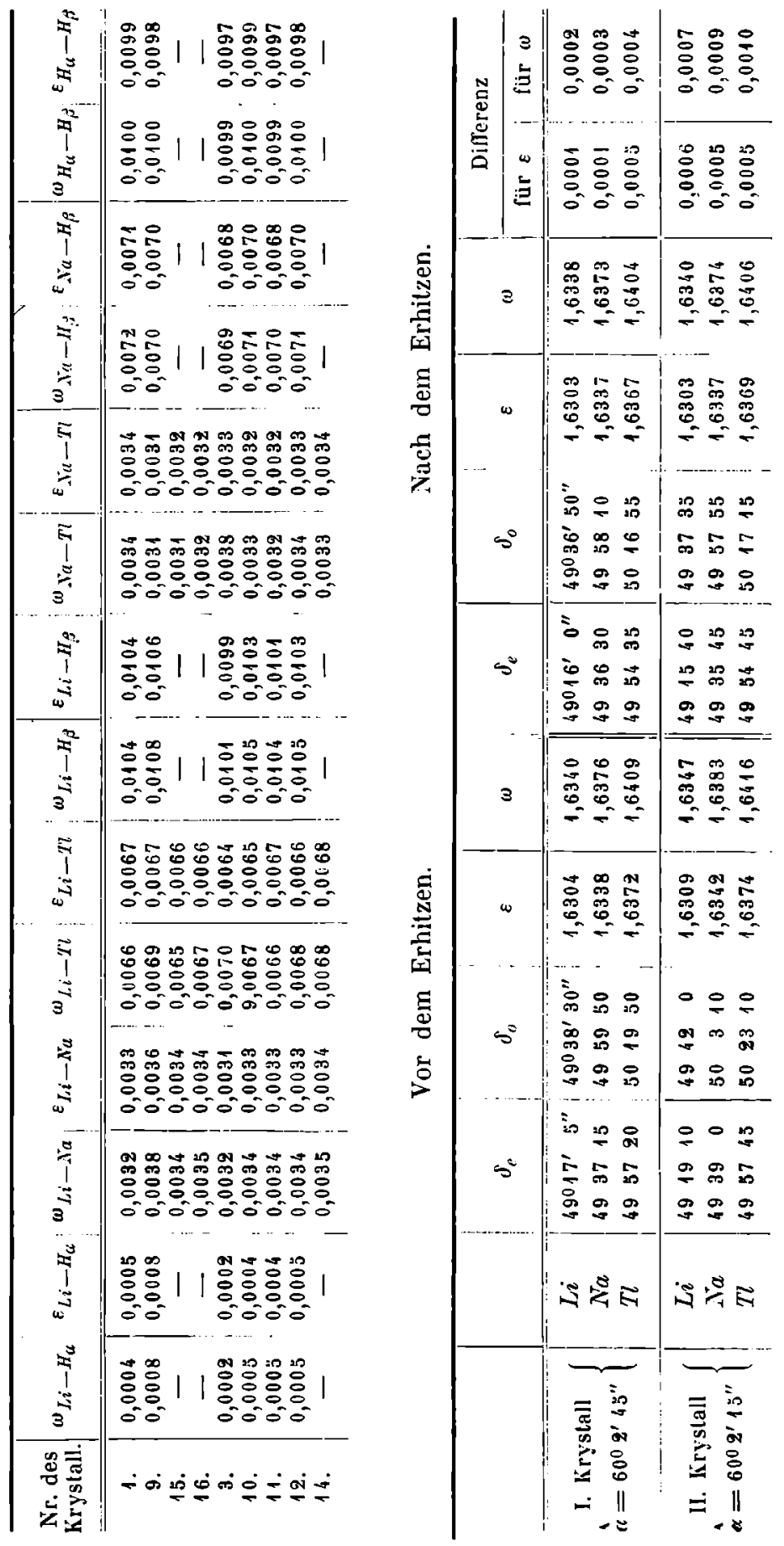


exponenten bestimmte ich von zwei Krystallen, und zwar bei ursprünglicher grüner Färbung und nach der durch Erhitzen erfolgten Entfürbung bei $\mathrm{Li}$-, $\mathrm{Na}$ - und $\mathrm{Tl}$-Licht. Der I. Krystall $(7 \mathrm{~mm}$ lang, $3 \mathrm{~mm}$ breit) hatte eine sehr lichte grünlichgelbe Farbe, war vollkommen klar ohne Sprünge oder Einschlüsse, an dem frei ausgebildeten Ende mit einigen Krystallflächen. Der II. Krystall (7 mm lang, $4 \mathrm{~mm}$ breit) war nicht gleichmässig gefärbt, an dem einen Ende lebhaft grünlich gelb, an dem anderen lichtgrün und hier auch mit Spaltungsrichtungen und Hämatiteinschlüssen; zur optischen Untersuchung diente der intensiv gefärbte Theil des Krystalles, die übrigen Flächen wurden mit schwarzer Farbe verdeckt. Die zwei Krystalle erhitzte ich einzeln im Platintiegel zwischen Sand gebettet unter grosser Vorsicht langsam bis zu starker Rothgluth. Nach ungefähr zwei Stunden hatten die Krystalle eine lichtgelbe Farbe angenommen, und zwar der I. Krystall nach kurzem, der II. nach lïnger dauerndem Erhitzen, jener wurde noch $1 \frac{1}{4}$, der letztere noch 2 Stunden lang stark erhitzt, wodurch sie vollkommen wasserklar wurden. An jedem wurden zwei beinahe tadellos reflectirende Prismenflächen ausgewählt und so vor, wie auch nach der Entfïrbung an denselben Partieen die Beobachtungen ausgeführt, was auch die goniometrische Messung des brechenden Winkels bestätigte.

In den entfärbten Krystallen war die Lichtabsorption geringer für beide Strahlen, die Intensität des ordentlichen und ausserordentlichen Strahles war noch bemerkbar verschieden, jedoch nicht so auffallend als bei der ursprünglichen Färbung der Krystalle; bei diesen konnte ich die abgelenkten Strahlen nur mit dem schwächeren Fernrohre scharf einstellen, hingegen nach der Entfärbung das stärkste Fernrohr mit dem Ramsden'schen Ocular benutzen. Die Resultate meiner Beobachtungen sind in der Tabelle auf S. 517 unten zusammengestellt.

Die Lichtbrechung beider Krystalle wurde nach dem Erhitzen schwächer, bei den hell gefärbten ist der Unterschied sehr klein — jedoch für die grünen Strahlen grösser - und liegt innerhalb der Fehlergrenzen. Bei den lebhaft gefärbten ist die Veränderung schon grösser $(0,0005-0,0040)$, und für grün ebenfalls grüsser als für roth und gelb. $\mathrm{Zu}$ bemerken ist noch, dass in beiden Krystallen die Lichtbrechung nach dem Entfärben für den ordentlichen Strahl auffallender geschwächt wurde als für den ausserordentlichen. Auffallend ist es, dass die verschiedene Lichtbrechung der Krystalle nach der Entfärbung so zu sagen vollkommen identisch wurde, der Unterschied ist 1-2 Einheiten der vierten Decimale. Die Mittelwerthe der Brechungsexsponenten der zwei entfärbten Krystalle wären:

\begin{tabular}{llll} 
& \multicolumn{1}{c}{$\omega$} & \multicolumn{1}{c}{$\varepsilon$} & \multicolumn{1}{c}{$\omega-\varepsilon$} \\
$\mathrm{Li}$ & 1,6339 & 1,6303 & 0,0036 \\
$\mathrm{Na}$ & 1,63735 & 1,5337 & 0,00365 \\
$\mathrm{Tl}$ & $1,640 \mathrm{3}$ & 1,6368 & 0,0037
\end{tabular}


Natürlicher Weise darf man keine allgemeinen Folgerungen ziehen von Resultaten, welche bloss an zwei Krystallen erhalten wurden, bevor noch an anderen $\Lambda$ patiten ähnliche Beobachtungen nichl gemacht wurden. Der Pleochroïsmus ist an allen Krystalleu auflallend, bei den dunkleren stärker als bei den lichteren, am schwächsten ist derselbe bei den blassgrünen und zwar
$o$ lichtgelb
$e$ bläulich aquamaringr'ün.

Die lebhaft grün gefürbten Krystalle wie $\mathrm{Nr}$. 9, 15 und 16 zeigen die Axenfarben

$$
\begin{aligned}
& o \text { rüthlichgelb } \\
& e \text { błäulichgrün. }
\end{aligned}
$$

Den stärksten Pleochroïsmus besitzen die gelblichen und bräunlichen Krystalle und zwar:

$$
\begin{aligned}
& o \text { brüunlichgelb } \\
& e \text { gelblichgrün. }
\end{aligned}
$$

Die grünlichgelben Krystalle zeigen dieselben $\Lambda$ xenfarben, nur nicht so lebhaft. An einem theilweise durchsichtigen, etwa $11 \mathrm{~mm}$ dicken dunkelgelben Krystalle war $e$ dunkelgrün ühnlich der Farbe mancher Turmaline, $o$ intensiv gelblichbraun; auch war die stïrkere Absorption ${ }^{1)}$ des ausserordentlichen Strahles auffallend.

Im convergent polarisirten Lichte ist an manchen Krystallen und geschliffenen Platten das Axenbild etwas gestürt, indem bei Drehung derselben um die optische Axe das schwarze Kreuz sich ein wenig öfnet. An zwei Platten, welche ich von einem gelblichgrünen und einem lichtgrünen Krystalle schliff, waren weder Feldertheilung noch ein schalenfürmiger Aufbau bemerkbar, wie dies an den violblauen Krystallen Mallardz), Dölter ${ }^{3}$ ) und Wolff ${ }^{4}$ ) beobachteten.

Herrn Hofrath Prof. J. Al. Krenner spreche ich auch hier meinen besten Dank aus für die Erlaubniss, dass ich meine Untersuchungen im mineralogisch-petrographischen Institule der Budapaster Universität ausführen konnte.

Budapest, im März 1904.

1) Nach Rosenbusch ist die stärkere Absorption des ausserordentlichen Stralıles selbst an dem farblosen Apatit bemerkbar (Mikroskop. Physiogr. d. petrogr. wichtistgen Mineralien 1892, lll. Aufl., 409\%, was auch ich an den durch Erhitzen entfärbten Krystallen beobachtete.

2) $\Lambda$ nnales d. Mines 1876 (7), 10, 147. Ref. diese Zeitschr. 1877, 1, 318.

3) N. Jahrb. f. Mineral. u.s. w. 1884, 2, 220. Ref. diese Zeitschr. 1886, 11, 333 .

4) Diese Zeitschr. 1902, 36, 443. 